\title{
Short-Term Results of Laparoscopic Radiofrequency Ablation Using a Multipolar System for Localized Hepatocellular Carcinoma
}

\author{
Naoki Morimoto Norio Isoda Yoshinari Takaoka Takuya Hirosawa \\ Shunji Watanabe Toshiya Otake Kozue Murayama Takeshi Fujieda \\ Mamiko Tsukui Natsumi Miyata Kohei Ono Shota Yamaguchi \\ Hironori Yamamoto \\ Division of Gastroenterology and Hepatology, Department of Internal Medicine, Jichi Medical \\ University, Tochigi, Japan
}

\author{
Keywords \\ Hepatocellular carcinoma $\cdot$ Radiofrequency ablation · Outcome $\cdot$ Surgery $\cdot$ Local ablation \\ therapy
}

\begin{abstract}
Background and Aim: Multipolar radiofrequency ablation (RFA) is feasible for the treatment of hepatocellular carcinoma (HCC) for which a large ablative area is planned, and it imposes a light physical burden on patients. Multipolar RFA via the percutaneous approach is performed in the majority of cases, but the efficacy of multipolar RFA with a laparoscopic approach has rarely been studied. This study aimed to evaluate the efficacy and safety of multipolar laparoscopic RFA (LRA) for localized HCC over the short term. Methods: From January 2014 to January 2016, 77 consecutive patients with 130 HCCs treated by multipolar LRA were assessed. One to three bipolar needle applicators were inserted under laparoscopic ultrasonography guidance, regardless of tumor location. We intended to achieve parallel insertions and no-touch ablation as much as possible. Results: The median size of the main tumor was $22 \mathrm{~mm}$ (range, 10-42 mm). The median follow-up time was 13.6 months (range, 3.1-24.8 months). In all cases, a sufficient ablative area was obtained as planned, without thermal injury of adjacent organs. During the follow-up period, all patients were alive with no local tumor progression, while intrahepatic recurrence distant from the primary site occurred in 7 patients. The 2-year local tumor progression-free survival rate and overall cancer-free survival rate were 100 and $81.6 \%$, respectively. There were no procedural major complications caused prolonging the hospitalization, and all patients were discharged without subjective symptoms 4-7 days after LRA. Conclusions: Multipolar LRA was efficacious in the treatment of localized HCCs by safely achieving a good ablative area.




\section{Liver Cancer}

\section{Introduction}

Hepatocellular carcinoma (HCC) is the sixth most common cancer and the most frequent cause of death in patients with cirrhosis [1]. In the selection of therapeutic options, liver function impaired by underlying chronic liver disease is one of the most important factors, in addition to tumor size and number [1,2]. According to the Barcelona clinic liver cancer staging classification, resection, liver transplantation, and ablation therapy are recommended as curative treatments for cases in the very early or early stage that consist of a single nodule or up to 3 nodules less than $3 \mathrm{~cm} \mathrm{[3,4].} \mathrm{Surgical} \mathrm{resection} \mathrm{is} \mathrm{considered} \mathrm{the} \mathrm{first-choice} \mathrm{treatment,}$ but its use is limited to the minority of cases due to underlying liver dysfunction [5]. Transplantation has become a favored option for HCC treatment, but the dearth of donor grafts is a problem in Japan. Radiofrequency ablation (RFA) is widely used worldwide as the treatment for localized small HCC because of its good local therapeutic effect with minimal invasion [6-8].

Osaki et al. [9] reported that multipolar RFA (CelonPOWER System; Olympus Medical Systems, Tokyo, Japan) was a device that was able to obtain a large ablative area safely in a short time, and it was approved for use in Japan in 2012.

We have performed laparoscopic RFA (LRA) for the treatment of HCC since 1999 and have performed LRA using a multipolar RFA system since 2014. In the current study, the short-term efficacy and safety of multipolar LRA were assessed.

\section{Materials and Methods}

\section{Patients}

The criteria for the use of RFA in our institution were Child-Pugh grade A or B, within the Milan criteria (1 lesion $\leq 5 \mathrm{~cm}$, or 2-3 lesions each $\leq 3 \mathrm{~cm}$ ). We performed RFA with laparoscopic approach in all cases, except in patients with severe intraperitoneal adhesion or intolerance for general anesthesia.

In this study period, regardless of tumor location, whether on the hepatic surface or deep inside the liver, we intended to perform multipolar LRA as the first-line procedure. Exclusion criteria for using multipolar RFA were cases in which (1) adhesions or adjacent major vessels caused impossibility for plural insertion surrounding the tumor or (2) the diameter of the main tumor was less than $15 \mathrm{~mm}$. In such cases, monopolar RFA was planned to be performed before operation.

From January 2014 to January 2016, 152 patients were diagnosed with HCCs and considered to have an indication for RFA. Eleven patients underwent percutaneous RFA caused by postoperative adhesions and in 50 patients, LRA using monopolar RFA applicator was performed according to the above criteria. Ninety-one patients were supposed to be treated by LRA using a multipolar RFA system. In 3 of them, treatment was changed to percutaneous RFA or surgical resection because of severe intraperitoneal adhesions. Of the remaining 88 patients, 77 consecutive patients with 130 HCCs with a follow-up of at least 3 months after LRA were enrolled in this study. The baseline clinical characteristics of the patients and nodules are shown in Table 1.

The study included 63 male and 14 female patients with a median age of 68 years (range, 37-88 years). Thirty-nine cases had a solitary tumor, and the other 38 cases had multiple HCCs. Sixty-eight patients were classified as Child-Pugh grade A, and 9 patients were classified as Child-Pugh grade B. Six out of 77 patients were positive for hepatitis B surface antigen, 51 patients were positive for hepatitis $C$ virus antibody, and the remaining 20 patients were negative for both hepatitis B surface antigen and hepatitis $\mathrm{C}$ virus antibody. The number of patients who had serum $\alpha$-fetoprotein concentrations $<100,100-400$, and $>400 \mathrm{ng} / \mathrm{dL}$ was 64,7 , and 6 , respectively. The number of patients who had serum des- $\gamma$-carboxyprothrombin concentrations $<100$, 100-400, and $>400 \mathrm{mAU} / \mathrm{mL}$ was 64,9 , and 0 , respectively. The serum des- $\gamma$-carboxyprothrombin level was not measured in 4 patients because they were on warfarin.

In 3 cases, LRA was performed after transcatheter arterial chemoembolization premeditatedly, and the remaining cases were treated by LRA alone. 


\section{Liver Cancer}

Table 1. Baseline characteristics of the patients undergoing multipolar laparoscopic radiofrequency ablation for hepatocellular carcinoma (77 patients, 130 tumors)

\begin{tabular}{l|l}
\hline Liver Cancer 2017;6:137-145 \\
\hline DOI: 10.1159/000450925 & $\begin{array}{l}\text { @ 2016 S. Karger AG, Basel } \\
\text { www.karger.com/lic }\end{array}$ \\
\hline
\end{tabular}

Morimoto et al.: Short-Term Results of Laparoscopic Radiofrequency Ablation Using a Multipolar System for Localized Hepatocellular Carcinoma

\begin{tabular}{|c|c|}
\hline Variable & Median (range) or $n(\%)$ \\
\hline Age, years & $68(37-88)$ \\
\hline Males & $63(81.8)$ \\
\hline \multicolumn{2}{|l|}{ Viral infection } \\
\hline HBsAg positive & $6(7.8)$ \\
\hline HCVAb positive & $51(66.2)$ \\
\hline Both negative & $20(26.0)$ \\
\hline Ascites & $12(15.6)$ \\
\hline Albumin, g/dL & $3.9(2.6-5.1)$ \\
\hline Total bilirubin, mg/dL & $0.85(0.37-2.55)$ \\
\hline Prothrombin time, \% & $84.4(50.4-119.9)$ \\
\hline Platelet count, $\times 10^{4} / \mu \mathrm{L}$ & $8.1(3.7-23.4)$ \\
\hline \multicolumn{2}{|l|}{ Child-Pugh class } \\
\hline A & $68(88.3)$ \\
\hline $\mathrm{B}$ & 9 (11.7) \\
\hline $\mathrm{C}$ & $0(0.0)$ \\
\hline \multicolumn{2}{|l|}{ Treatment experience } \\
\hline Treatment naïve & $47(61.0)$ \\
\hline Recurrent case & $21(39.0)$ \\
\hline \multicolumn{2}{|l|}{ Diagnosis } \\
\hline Pathology & $49(63.6)$ \\
\hline Imaging & $28(36.7)$ \\
\hline Diameter of main tumor, mm & $22(10-42)$ \\
\hline \multicolumn{2}{|l|}{ Number of tumors } \\
\hline 1 & $39(50.6)$ \\
\hline 2 & $24(31.2)$ \\
\hline$\geq 3$ & $14(18.2)$ \\
\hline \multicolumn{2}{|l|}{ Location of tumor } \\
\hline Left lobe & $41(31.5)$ \\
\hline Right anterior lobe & $44(33.8)$ \\
\hline Right posterior lobe & $44(33.8)$ \\
\hline Caudate lobe & $1(0.8)$ \\
\hline Serum AFP, ng/dL & $12(1-5,508)$ \\
\hline Serum DCP, mAU/mL & $24(9-316)$ \\
\hline
\end{tabular}

HBsAg, hepatitis B surface antigen; HCVAb, hepatitis C virus antibody; AFP, $\alpha$-fetoprotein; DCP, des- $\gamma$-carboxyprothrombin.

Forty-nine patients were diagnosed as HCC by pathological findings, and the other 28 were diagnosed based on typical radiographic findings of the tumor showing early-phase enhancement followed by latephase wash-out of contrast agent on computed tomography (CT) and/or magnetic resonance imaging.

Before enrollment and any procedures were undertaken, written informed consent was obtained from all patients. The study was approved by the Institutional Review Board of Jichi Medical University Hospital and was in accordance with the principles of the Declaration of Helsinki and Good Clinical Practice.

\section{Multipolar RFA}

Two electrodes across the insulator are located at the tip of a 15-gauge, 25-cm-long, internally cooled needle-type applicator. One to three applicators can be used simultaneously. Radiofrequency current flows between all possible combinations of electrodes with an automatic impedance control, and a homogeneous ablative area is formed. The sizes of the electrodes and the numbers of applicators are selected according to planning for the expected ablative area. Generally, the energy amount followed the manufacturer's recommendation, and parallel insertion of multiple applicators is recommended to obtain the planned ablative area. 


\section{Liver Cancer}

Fig. 1. Scheme of the immersion method. In some cases, poor visualization of the tumor occurs due to tumor location or hepatic surface irregularity (a, b). By infusion of saline solution and making the space (immersion method), a good echo window is obtained, and thermal injury of adjacent organs is also avoided during ablation therapy $(\mathbf{c}, \mathbf{d})$.

\begin{tabular}{l|l}
\hline Liver Cancer 2017;6:137-145 \\
\hline DOI: 10.1159/000450925 & $\begin{array}{l}\text { @ 2016 S. Karger AG, Basel } \\
\text { www.karger.com/lic }\end{array}$ \\
\hline
\end{tabular}

Morimoto et al.: Short-Term Results of Laparoscopic Radiofrequency Ablation Using a Multipolar System for Localized Hepatocellular Carcinoma

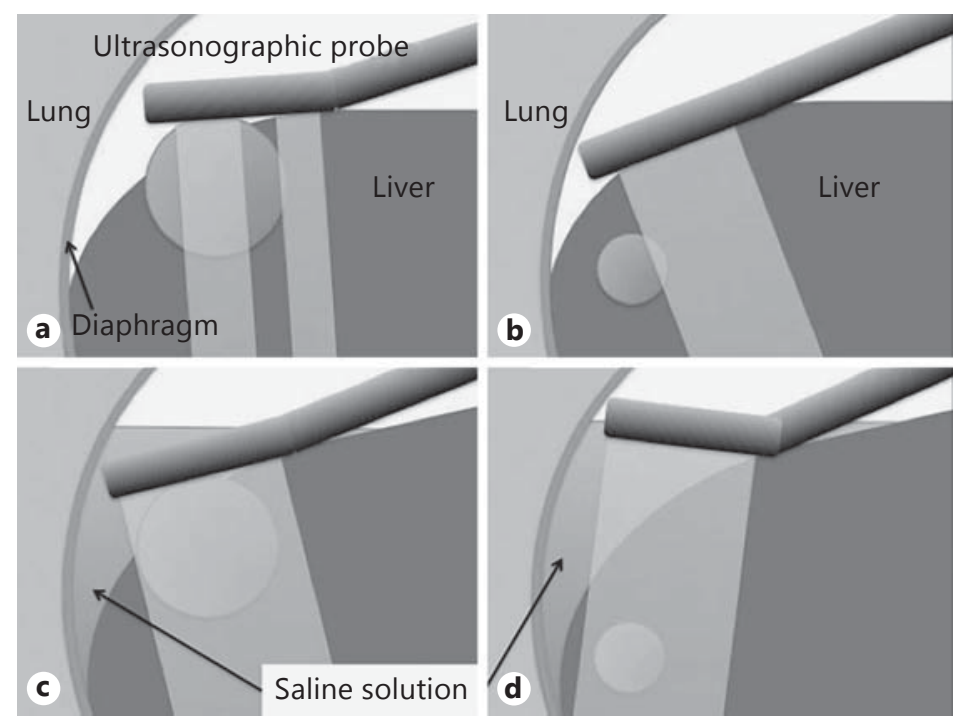

\section{Laparoscopic RFA}

LRA was performed under general anesthesia. Carbon dioxide was used for pneumoperitoneum, and the pressure of pneumoperitoneum was kept below $10 \mathrm{~mm} \mathrm{Hg}$ with the use of an automatic insufflator. The first trocar with a diameter of $11 \mathrm{~mm}$ was inserted into the upper left umbilical region. After the appearance of the liver and the intraperitoneal space were observed by laparoscopy, a 12-mm port for ultrasonography and a 5 -mm port for assistant forceps were inserted. The tumors and the vicinity were examined in detail by laparoscopic ultrasonography with a linear-type probe, and then the safe route of insertion that would avoid injury to the vessels was decided.

A 21-gauge guiding needle was inserted near the target under ultrasonographic guidance and laparoscopic visualization. A 13-gauge metallic sheath was inserted along the guiding needle, and the radiofrequency applicator was placed in the planned position accurately with fine adjustment.

By a change of body position and infusion of saline solution to make the fluid space (immersion method), not only a good echo window is obtained, but also thermal injury of adjacent organs is avoided during ablation therapy (Fig. 1).

In the treatment of tumors $\leq 2 \mathrm{~cm}$, no-touch ablation was performed where electrodes were not inserted into the tumor but only in the periphery surrounding the tumor. In the treatment of tumors $>2 \mathrm{~cm}, 3$ applicators were placed in parallel near the lateral border of the tumor, and overlapping ablations were added as needed. Needle tract cauterization was performed to prevent bleeding and seeding of malignant cells in all sessions.

Coagulation parameters were assessed in all patients, and in cases of platelet counts less than 50,000/ $\mu \mathrm{L}$, platelet transfusions were performed before LRA.

\section{Evaluation of Therapeutic Efficacy and Complications}

Dynamic CT was carried out 4 or 5 days after LRA. The ablative area was assessed and it was examined whether any major complications such as intraperitoneal bleeding, injury of adjacent organs, pleural effusion, and ascites were present.

After the patient's discharge, dynamic CT or magnetic resonance imaging and blood examinations including $\alpha$-fetoprotein and des- $\gamma$-carboxyprothrombin were performed every 3 months. Local tumor progression was defined as the appearance of a viable cell nest touching the original tumor, and distant recurrence was defined as the emergence of new $\operatorname{HCC}(\mathrm{s})$ separate from the primary site. 


\section{Liver Cancer}

Fig. 2. Cumulative recurrencefree survival rates. There was no locoregional recurrence and mortality. The 2-year cumulative local tumor progression-free survival rate is $100 \%$. Intrahepatic recurrence distant from the primary site was observed in 7 patients, and the overall cancer-free survival rates are $90.9 \%$ at 1 year and $81.6 \%$ at 2 years.

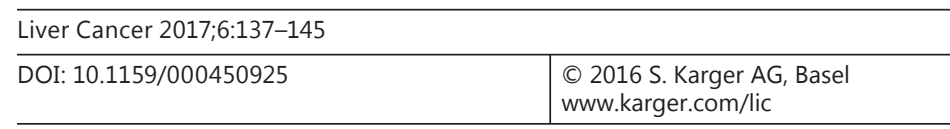

Morimoto et al.: Short-Term Results of Laparoscopic Radiofrequency Ablation Using a Multipolar System for Localized Hepatocellular Carcinoma

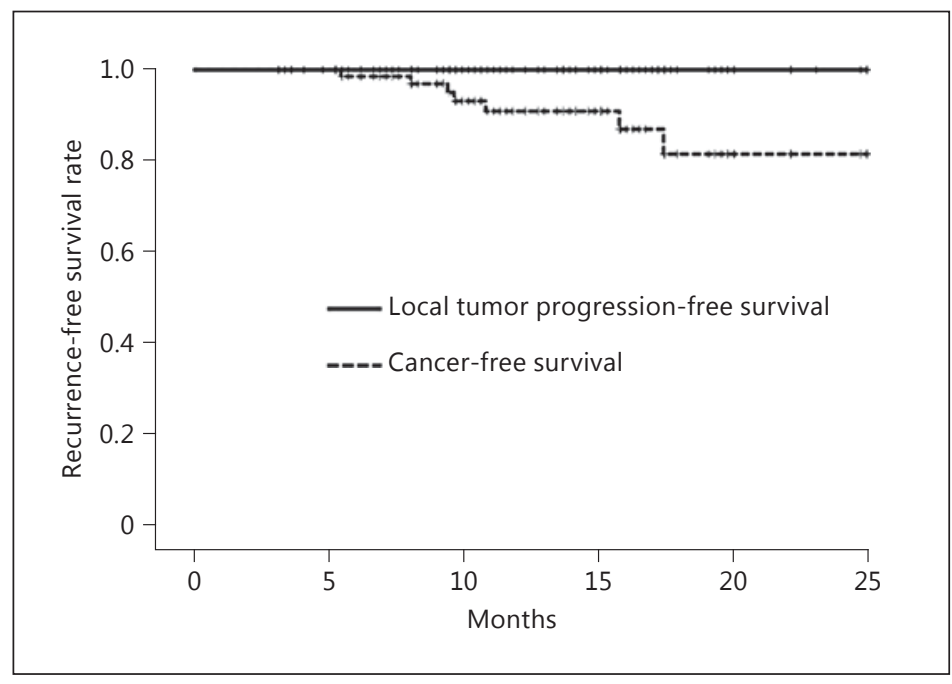

\section{Results}

This study evaluated 77 patients with 130 lesions. The median size of the main tumor was $22 \mathrm{~mm}$ (range, 10-42 mm). Fifty-eight out of 130 tumors were located in the hepatic surface and the remaining 72 tumors were located deep inside the liver. The number of tumors in segments $1-8$ was $1,8,19,14,11,19,25$ and 33 , respectively. All main tumors were treated with multiple applicators, with 21 nodules ablated by 2 applicators and the other 59 nodules treated using 3 applicators. The ablative time was $13.2 \mathrm{~min}$ (range, 3-42 $\mathrm{min}$ ) per nodule. The median operative time was $138.5 \mathrm{~min}$ (range, 83-299 min), and that of solitary HCC cases was 121 min (range, 83-185 min).

In all cases, sufficient ablative areas were obtained as planned, without thermal damage to adjacent organs on CT performed 4 or 5 days after LRA. The median follow-up time was 13.6 months (range, 3.1-24.8 months). During the follow-up period, all patients were alive with no local tumor progression, though 7 patients developed intrahepatic recurrences distant from the primary site. The 2-year cumulative local tumor progression-free survival rate was $100 \%$, and the overall cancer-free survival rates were $90.9 \%$ at 1 year and $81.6 \%$ at 2 years (Fig. 2). Four of seven patients who developed distant recurrence were cases with 3 lesions originally. Of the 7 cases, LRA was performed for the recurrent tumor in 3 cases. Surgical resection was carried out in one case because the recurrent tumor was located beneath the heart and an adhesion between the target lesion and diaphragm was present. Three cases with multiple recurrences were treated with transcatheter arterial chemoembolization.

In almost all patients, transaminase levels were elevated to around $300 \mathrm{IU} / \mathrm{L}$ transiently, but they improved quickly with conservative follow-up. Seventeen patients had a fever of $38-39^{\circ} \mathrm{C}$ after LRA, but only 9 cases required administration of antipyretics, and no infectious complications, including an abscess, occurred. Although minimal pleural effusion and ascites near the therapeutic site were present after the procedure in 5 and 6 cases, respectively, they improved without administration of diuretics in all cases. Postoperative intraperitoneal bleeding did not occur, but a small amount of bile juice oozed from the needle tract of the liver in one case. Because the ooze was observed just after needle removal, cauterization and suturing to the liver surface were performed laparoscopically, and then the leakage of bile was getting lesser but continued. Therefore, endoscopic retrograde biliary drainage was 
Morimoto et al.: Short-Term Results of Laparoscopic Radiofrequency Ablation Using a Multipolar System for Localized Hepatocellular Carcinoma

performed immediately after completion of LRA, and the patient left the hospital 7 days after the procedures without any symptoms.

All patients were discharged 4-7 days after LRA without deviation from the clinical pathway.

\section{Discussion}

RFA plays an important role in localized small HCC therapy. Approaches include percutaneous, laparoscopic, and open RFA. Despite a lack of prospective comparative studies, there are reports of good outcomes using LRA [10-13]. Altering the patient's body position and injecting saline solution (immersion method) not only allows good tumor visualization on ultrasonography, but also enables the physical distance between the lesion and surrounding organs such as the diaphragm and gastrointestinal tract to be maintained, thus preventing thermal injury to these organs during treatment [13-15].

LRA can be performed in virtually all areas of the liver regardless of tumor location, and it is particularly useful in treating hepatic surface HCC. In addition to preventing thermal burns to the abdominal wall and other surrounding organs, LRA enables treatment from outside the border of the tumor while avoiding direct puncture precisely for the prevention of seeding of malignant cells with a combination of ultrasonographic and laparoscopic observation $[16,17]$.

A sure hemostasis can be achieved using a coagulation forceps under laparoscopic observation in case of any bleeding from the abdominal wall or the liver. In this study, no postoperative bleeding complication occurred, and LRA was considered to be a safe and reliable procedure. There was, however, one case of persistent bile leakage from the liver puncture route. Slight oozing persisted during the suture procedure, so endoscopic biliary drainage was performed promptly after LRA, and then the patient was subsequently discharged without any worsening of signs or symptoms 7 days after LRA. While the laparoscopic procedure alone was not adequate to complete the treatment, laparoscopic confirmation of the patient's intraperitoneal condition and prompt response contributed to the safe outcome.

The CelonPOWER multipolar RFA system approved for use in Japan enables simultaneous insertion of up to 3 bipolar electrode applicator needles. No ground pad is required, so it does not cause thermal injury to the skin [9], and the system can also be used safely in patients with cardiac pacemakers [18]. One of the greatest advantages of the multipolar system is its ability to achieve a large ablative area in a short period of time as planned, because it is not prone to a vascular heat sink effect that would result in unexpected reduction of the ablative area $[19,20]$. In the present study, a good therapeutic area was also achieved with an ablative time of 30-40 min, even when using the overlap method to treat tumors of $\geq 4 \mathrm{~cm}$. The short ablative time decreases patient burden; thus, multipolar RFA is a suitable procedure for the treatment of large tumors exceeding $3 \mathrm{~cm}$.

Moreover, no-touch ablation, which involves locating the applicator so as to surround the margin of the tumor without direct puncture, is expected to prevent tumor dissemination and local progression [21, 22].

No-touch ablation is a useful procedure for preventing seeding, and it is particularly crucial for treating hepatic surface lesions. However, RFA using the monopolar applicator to pierce the tumor is reportedly associated with a local tumor progression rate of 3-19\% [6, 23-26]. In the case of lesions located deep inside the liver, a piercing of the tumor does not lead to local tumor progression, and a sufficient ablative safety margin is a significant factor related to the local tumor progression $[24,26]$. No-touch ablation could not be performed on large tumors in the present study due to limitations on the permitted needle distance, and it 

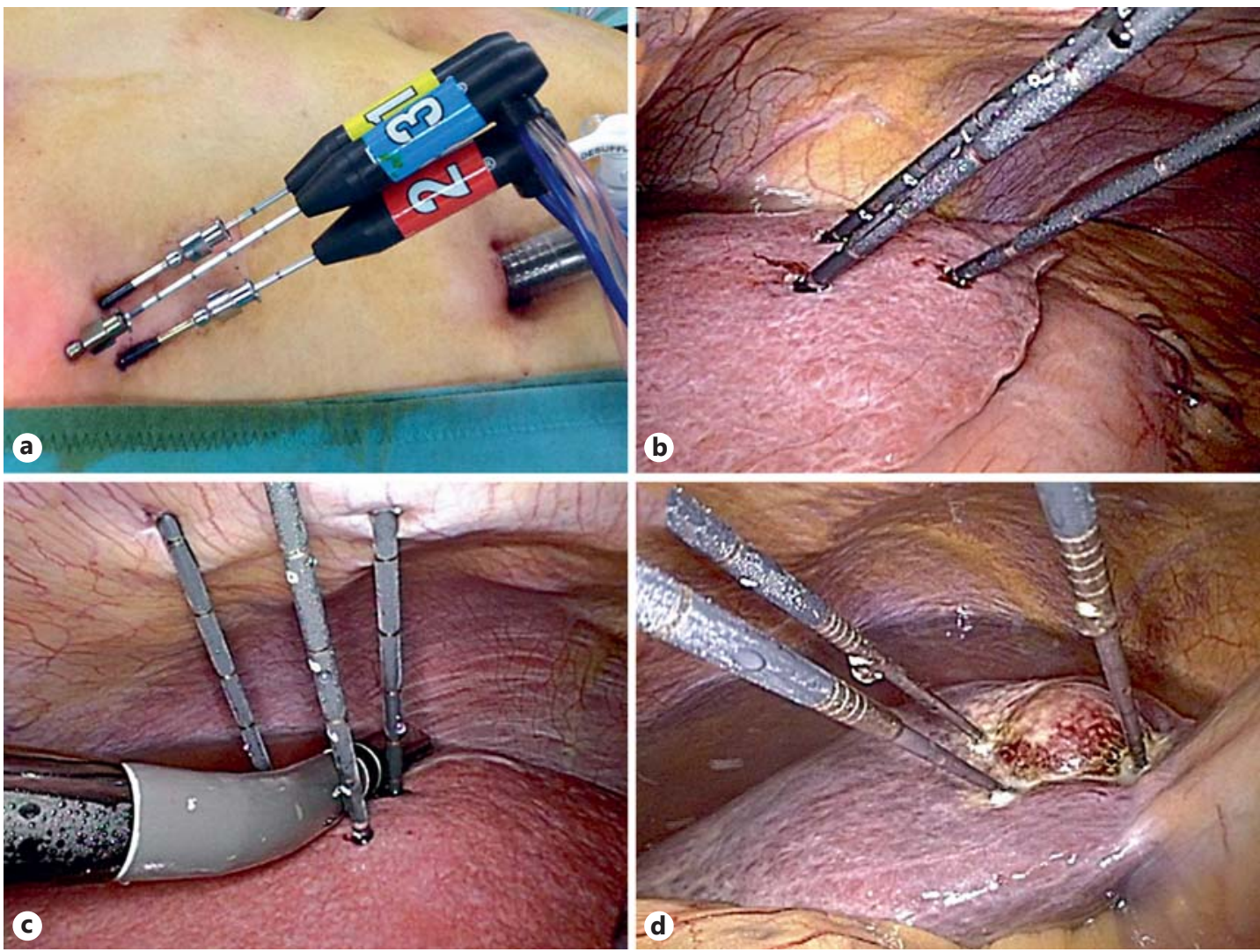

Fig. 3. Multipolar laparoscopic radiofrequency ablation. The laparoscopic approach enables parallel insertion of multiple applicators without limitation of rims, irrespective of tumor location (a-d). Precise needle placement is performed under ultrasonography guidance with a linear-type probe (c). Especially in cases of a tumor on the hepatic surface, multipolar laparoscopic radiofrequency ablation is highly useful to perform no-touch ablation and avoid thermal damage to adjacent organs (d).

was only performed on tumors approximately $\leq 2 \mathrm{~cm}$. Although the study results are shortterm, there has been no local tumor progression in any of the patients. Even without the no-touch procedure, surrounding the tumor as much as possible by making a marginal puncture might lead to achievement of a sufficient ablative area with a safety margin.

Multipolar RFA tends to yield a long and narrow ablative area with a single applicator, and a therapeutic area with a flat, short cross-section can also be achieved with 2 applicators [27]. The simultaneous use of 3 applicators provides a good sphere-like ablative area. Therefore, achieving a sufficient ablative area covering the tumor with an adequate safety margin as per the treatment plan requires the parallel insertion of 3 applicators wherever feasible. The laparoscopic approach offers parallel insertion of multiple applicators into most areas of the liver without limitation caused by the ribs and/or the echo window. It makes the laparoscopic approach a suitable procedure for leveraging the advantages of multipolar RFA (Fig. 3).

The use of multiple needles with greater thickness than a conventional monopolar applicator also means that multipolar RFA involves a slightly higher risk of bleeding from the needle tract when removing the needle, so it is useful to spend more time to achieve sufficient tract cauterization. The laparoscopic approach also prevents thermal burns to the abdominal wall when performing tract cauterization, and it can ensure hemostasis with the use of coag- 
ulation forceps in the event of bleeding, so it is also useful in terms of its ability to compensate for the disadvantages of multipolar RFA.

On the other hand, LRA cannot be performed in patients who are unable to undergo general anesthesia and those with severe abdominal adhesions, and it is also difficult to perform a safe and reliable therapy on patients with a tumor in Spiegel's lobe, so it is slightly limited in terms of its indications.

Limitations of this study include the fact that it was a retrospective cohort with a small patient population in a single institution. The short follow-up time might underestimate the likelihood of local tumor progression and complications. There was a slight bias in the selection of subjects due to the fact that monopolar RFA is typically selected when secure and safe insertion of multiple applicators is deemed to be infeasible as a result of intraperitoneal adhesions and the relationships of surrounding vessels.

This study was a non-comparative single-arm evaluation. The short-term results of multipolar LRA were relatively good; however, the laparoscopic approach required general anesthesia and long operation time. The approach methods or use of equipment would be selected depending on the conditions of tumor, patient and institution.

\section{Conclusion}

In this study, all patients who underwent LRA stayed alive without local tumor progression and major complication in short-term results. Multipolar LRA was efficacious in the treatment of localized HCCs by safely achieving a good ablative area. It is particularly effective in the treatment of lesions $\geq 3 \mathrm{~cm}$ and those on the liver surface that are close to the gastrointestinal tract, gallbladder, and other surrounding organs, and it therefore warrants consideration as a useful treatment option. Further study with a larger population is required to investigate long-term outcomes.

\section{Acknowledgements}

The authors thank Ms. Minori Kobori for the helpful secretarial assistance.

\section{Disclosure Statement}

The authors have no conflicts of interest or financial funding to report.

\section{References}

1 European Association for the Study of the Liver, European Organisation for Research and Treatment of Cancer: EASL-EORTC clinical practice guidelines: management of hepatocellular carcinoma. J Hepatol 2012;56:908943.

2 Kudo M, Matsui O, Izumi N, Iijima H, Kadoya M, Imai Y, et al: JSH Consensus-Based Clinical Practice Guidelines for the Management of Hepatocellular Carcinoma: 2014 Update by the Liver Cancer Study Group of Japan. Liver Cancer 2014;3:458-468.

3 Llovet JM, Di Bisceglie AM, Bruix J, Kramer BS, Lencioni R, Zhu AX, et al: Design and endpoints of clinical trials in hepatocellular carcinoma. J Natl Cancer Inst 2008;100:698-711.

4 Bruix J, Sherman M: Management of hepatocellular carcinoma: an update. Hepatology 2011;53:1020-1022.

5 Lai E, Fan S-T, Lo C-M, Chu K, Liu C, Wong J, et al: Hepatic resection for hepatocellular carcinoma. Ann Surg 1995;221:291-298.

6 Shiina S, Tateishi R, Arano T, Uchino K, Enooku K, Nakagawa H, et al: Radiofrequency ablation for hepatocellular carcinoma: 10-year outcome and prognostic factors. Am J Gastroenterol 2012;107:569-577. 
7 Salhab M, Canelo R: An overview of evidence-based management of hepatocellular carcinoma: a meta-analysis. J Cancer Res Ther 2011;7:463-475.

8 Wang JH, Wang CC, Hung CH, Chen CL, Lu SN: Survival comparison between surgical resection and radiofrequency ablation for patients in BCLC very early/early stage hepatocellular carcinoma. J Hepatol 2012;56: 412-418.

9 Osaki Y, Ikeda K, Izumi N, Yamashita S, Kumada H, Hatta S, et al: Clinical effectiveness of bipolar radiofrequency ablation for small liver cancers. J Gastroenterol 2013;48:874-883.

10 Santambrogio R, Barabino M, Bruno S, Costa M, Ceretti AP, Angiolini MR, et al: Long-term outcome of laparoscopic ablation therapies for unresectable hepatocellular carcinoma: a single European center experience of 426 patients. Surg Endosc 2016;30:2103-2113.

11 Hirooka M, Kisaka Y, Uehara T, Ishida K, Kumagi T, Watanabe Y, et al: Efficacy of laparoscopic radiofrequency ablation for hepatocellular carcinoma compared to percutaneous radiofrequency ablation with artificial ascites. Gastroenterol Endosc 2010;52:278-285.

12 Herbold T, Wahba R, Bangard C, Demir M, Drebber U, Stippel DL: The laparoscopic approach for radiofrequency ablation of hepatocellular carcinoma - indication, technique and results. Langenbecks Arch Surg 2013; 398:47-53.

13 Nagae G, Ido K, Isoda N, Sato S, Kita H, Sugano K: Laparoscopic ablation therapy for hepatocellular carcinoma. Dig Endosc 2005;17:1-8.

14 Ido K, Isoda N, Sugano K: Microwave coagulation therapy for liver cancer: laparoscopic microwave coagulation. J Gastroenterol 2001;36:145-152.

15 Kawamoto C, Ido K, Isoda N, Hozumi M, Nagamine N, Ono K, et al: Long-term outcomes for patients with solitary hepatocellular carcinoma treated by laparoscopic microwave coagulation. Cancer 2005;103:985993.

16 Tsuji K, Nishimori H, Sakurai Y, Mitsui S, Miyamoto N, Matsunaga T, et al: Efficacy and long-term outcome of laparoscopic microwave coagulation or radiofrequency ablation therapy for surface-type hepatocellular carcinoma. Dig Endosc 2004;16:117-121.

17 Morimoto N, Isoda N, Watanabe S, Otake T, Hirosawa T, Tsukui M, et al: A case of small hepatocellular carcinoma treated with laparoscopic multipolar radiofrequency ablation with a no-touch ablation procedure. Clin J Gastroenterol 2014;7:510-515.

18 Ikeda K, Osaki Y, Nakanishi H, Nasu A, Kawamura Y, Jyoko K, et al: Recent progress in radiofrequency ablation therapy for hepatocellular carcinoma. Oncology 2014;87:73-77.

19 Pillai K, Akhter J, Chua TC, Shehata M, Alzahrani N, Al-Alem I, et al: Heat sink effect on tumor ablation characteristics as observed in monopolar radiofrequency, bipolar radiofrequency, and microwave, using ex vivo calf liver model. Medicine (Baltimore) 2015;94:e580.

20 Al-Alem I, Pillai K, Akhter J, Chua TC, Morris DL: Heat sink phenomenon of bipolar and monopolar radiofrequency ablation observed using polypropylene tubes for vessel simulation. Surg Innov 2014;21:269-276.

21 Kawamura Y, Ikeda K, Fukushima T, Hara T, Hosaka T, Kobayashi M, et al: Potential of a no-touch pincer ablation procedure for small hepatocellular carcinoma that uses a multipolar radiofrequency ablation system: an experimental animal study. Hepatol Res 2014;44:1234-1240.

22 Seror O, N'kontchou G, Van Nhieu JT, Rabahi Y, Nahon P, Laurent A, et al: Histopathologic comparison of monopolar versus no-touch multipolar radiofrequency ablation to treat hepatocellular carcinoma within Milan criteria. J Vasc Interv Radiol 2014;25:599-607.

23 Gao J: Radiofrequency ablation for single hepatocellular carcinoma $3 \mathrm{~cm}$ or less as first-line treatment. World J Gastroenterol 2015;21:5287.

24 Hirooka M, Ochi H, Koizumi Y, Tokumoto Y, Hiraoka a, Kumagi T, et al: Local recurrence of hepatocellular carcinoma in the tumor blood drainage area following radiofrequency ablation. Mol Clin Oncol 2014;2:182186.

25 Kim Y, Lim HK, Rhim H, Lee MW, Choi D, Lee WJ, et al: Ten-year outcomes of percutaneous radiofrequency ablation as first-line therapy of early hepatocellular carcinoma: analysis of prognostic factors. J Hepatol 2013; 58:89-97.

26 Teng W, Liu K-W, Lin C-C, Jeng W-J, Chen W-T, Sheen I-S, et al: Insufficient ablative margin determined by early computed tomography may predict the recurrence of hepatocellular carcinoma after radiofrequency ablation. Liver Cancer 2015;4:26-38.

27 Rempp H, Mezger D, Voigtlaender M, Scharpf M, Hoffmann R, Pereira PL, et al: A comparison of internally water-perfused and cryogenically cooled monopolar and bipolar radiofrequency applicators in ex vivo liver samples. Acad Radiol 2014;21:661-666. 